\title{
MS16-02 | In Situ Methods for the Analysis of Functional Materials
}

Weidenthaler, Claudia (Max-Planck-Institut für Kohlenforschung, Mülheim an der Ruhr, GER)

Monitoring materials under non-ambient conditions opens up possibilities to gain detailed insights how materials behave under reaction conditions. The variety of analytical tools which are nowadays available for in situ or operando studies is as wide as the types of reactions which can be investigated. The combination of diffraction methods with spectroscopy enables deep insights into structural but also compositional changes during crystallization or chemical reactions. It will be shown that the use of in-house diffraction equipment can provide detailed insight into structure and microstructure changes. In situ diffraction data contain not only useful information on the averaged crystal structures but also on changes of local structures. The talk we will focus mainly on the design of in situ diffraction experiments performed under variable temperatures (between $100 \mathrm{~K}$ and $1000 \mathrm{~K}$ ), elevated gas pressures, adjustable reaction gas atmospheres, or mechanochemical reaction conditions. The materials which will be presented cover hydrogen storage materials as well as inorganic catalysts, or materials used for electrochemical applications. Hydrogenation studies performed with in-house diffractometers at variable temperatures and hydrogen pressures require the construction of tailor-made sample environments. Reactions with ammonia as hydrogen carrier are challenging due to experimental restrictions. Temperature dependent crystallization studies of Pt-based electrocatalysts from the precursors to the alloys represent a combination of crystal structure refinement and atomic pair distribution analysis. 